\title{
Two eccentric cylinders in a uniform electric field
}

\author{
A. S. Starkov, K. V. Korzenkov, K. A. Starkov \\ ITMO University, Kronverkskiy, 49, St. Petersburg, 197101, Russia \\ starkovalexandr@corp.ifmo.ru,korund607@yandex.com, ferroelectrics@ya.ru
}

PACS 77.84.Lf,77.22.d,65.80.g

DOI 10.17586/2220-8054-2017-8-2-277-281

The present study examines the electric field distribution in the structure made of two eccentric dielectric cylinders. In oder to find the potential
of electric field, we employ a bipolar coordinate system. The obtained results allow one to quantify the impact of the eccentricity on effective
characteristics of a periodic set of eccentric cylinders. Special attention is paid to the analysis of the structure polarizability.

Keywords: Eccentric cylinders, polarizability, Maxwell-Garnett approximation, effective dielectric constant.

Received: 11 December 2016

Revised: 23 February 2017

\section{Introduction}

Currently, composites are one of the most promising materials for use in new technologies [1,2]. The most widespread are laminates, fibrous (reinforcing component - the fibrous structure), and filled materials (the reinforcing component - particles). In turn, compared with laminated composites, cylindrical composites (fibers) have more compact structure, which is beneficial for device miniaturization [3,4]. Such composites are usually modeled by periodic sets of cylinders made of one material (filler) and placed in the second material (matrix). The calculation of effective parameters of the composite medium is based, as a rule, on the solution of the problem for the single cylinder (inclusion) in the surrounding external field and subsequent averaging procedure [5]. To be more precise, for the description of corresponding inclusions it is necessary to use the equations considering interactions of all possible fields: electromagnetic, elastic and thermal.

The surface and nonlocal effects start to play a significant role for the inclusions of size about one nanometer. Therefore, in general there is a need to solve the integro-differential equations. In our work, an average diameter of inclusions is assumed to be tens of nanometers and, thus, we can restrict ourselves by differential equations. Moreover, we analyze the case where the electrical properties of composites can be considered independently of the elastic ones. That is, it is sufficient to find the electric potential by means of the Laplace equation. In a classic work by Rayleigh [6], the electric potential was computed for a conducting composite consisting of a periodic array of inclusions (cylinders and spheres). More complex core-shell structures, which have appeared in recent years, allow one to improve the desired properties of composites. However, these structures complicate the determination of their properties even in the elementary cases [7-9].

Future prospects for the development of composites are associated with the finding of non-smooth fillings of untypical shapes. As already well known [9], the presence of peaks or edges of a filling leads to the appearance of singularities in the electric field that has a strong impact on the effective permittivity. The numerical calculation of such structures is not an easy task and requires skilled techniques for its solution (see, e.g. [9, 10]). In this study, we investigate the possibility to control the dielectric constant by changing the distance between the boundaries of the different layers (shells). We consider the electric potential in the medium consisting of cylindrical inclusion in which one more cylinder is placed (see, Fig. 1). In other words, two dielectric cylinders are considered. If the cylinders have no common points, then the solution of an electrostatic problem can be found in [9, 11]. Summing it up, this paper examines a cylinder completely immersed inside another one. A case of intersecting cylinders remains unexplored.

Note that due to the analogy between the stationary electric and thermal fields, the effective thermal capacity of a composite with inclusions in the form of eccentric cylinders can be determined by the same procedure.

\section{The solution of the general problem}

\subsection{The problem statement}

As a starting point, we consider an electric field in the system illustrated in Fig. 1. Inclusion consists of two eccentric circles of radii of $r_{1}$ and $r_{2}$ which centers are located at distance $d$ from each other. The surfaces of 


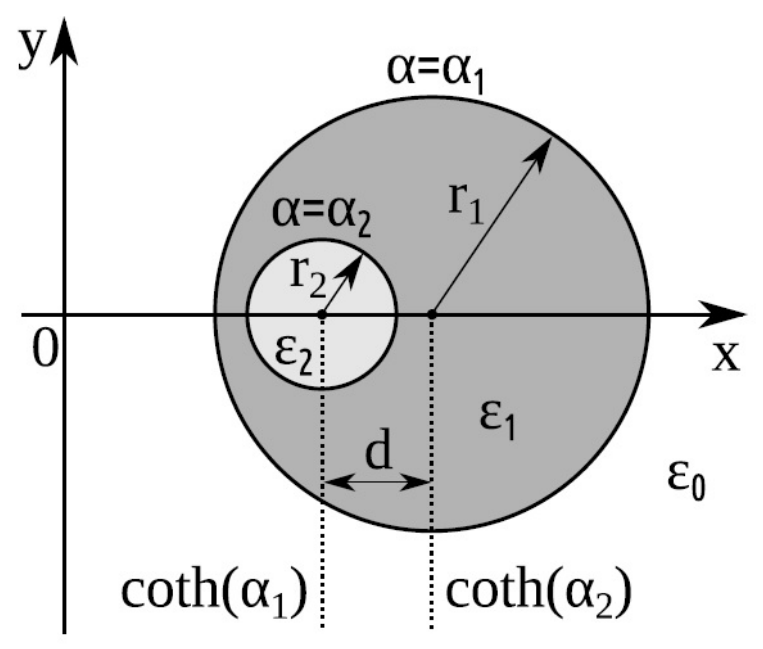

FIG. 1. The mutual arrangement of two dielectric cylinders

circles are $S_{1,2}$. To describe electrostatic field, we employ a Cartesian coordinate system $(x, y)$. By $\varepsilon_{1}, \varepsilon_{2}$ we denote dielectric permeability of circles and dielectric permeability of external space (matrix) by $\varepsilon_{0}$.

Similarly, we will use the subscripts 1,2 to identify quantities related to circles and subscript 0 for the matrix parameters. In the absence of any subscript, the expression is fulfilled in all environments. The intensity of the external electric field is supposed to be a constant vector of length $E$. In the solution of our problem it is necessary to find the potential $u$, which satisfies the two-dimensional Laplace equation:

$$
\Delta u=0,
$$

and is continuous at the interfaces $S_{1,2}$ together with the normal component of the electric displacement:

$$
u_{0}\left|S_{1}=u_{1}\right| S_{1},\left.\quad \varepsilon_{1} \frac{\partial u_{0}}{\partial n}\right|_{S_{1}}=\left.\varepsilon_{2} \frac{\partial u_{1}}{\partial n}\right|_{S_{1}}, \quad u_{1}\left|S_{2}=u_{2}\right| S_{2},\left.\quad \varepsilon_{1} \frac{\partial u_{1}}{\partial n}\right|_{S_{2}}=\left.\varepsilon_{2} \frac{\partial u_{2}}{\partial n}\right|_{S_{2}} .
$$

Moreover, at the distance far from the inclusion, the potential $u$ tends to the potential of the external field:

$$
u_{0} \rightarrow-E_{0}(x \cos \gamma+y \sin \gamma) .
$$

Here, $n$ is normal to the surfaces $S_{1,2}, E_{0}$ is the electric stress, and $\gamma$ is an angle between the external field and the $x$-axis. Because of the lack of symmetry, the solution of Eqs. (1)-(3) depends on the angle $\gamma$.

If we introduce the potential $U$ that tends to $U_{0}=x+\mathrm{i} y$ at large distances from the inclusion, then the desired potential $u$ can be expressed in terms of $U$ as:

$$
u=-E_{0}(\operatorname{Re} U \cos \gamma+\operatorname{Im} U \sin \gamma) .
$$

Further, it is reasonable to switch to a bipolar coordinate system $(\alpha, \beta)[12]$ associated with the Cartesian by the relations:

$$
x+i y=c \tanh \left(\frac{\alpha+i \beta}{2}\right) .
$$

If we define the parameters $\alpha_{1}, \alpha_{2}$ and $c$ from:

$$
\cosh \alpha_{j}=\frac{r_{1}^{2}-r_{2}^{2}+(-1)^{j} d^{2}}{2 r_{j} d}, \quad c=r_{1} \sinh \alpha_{1},
$$

then the circles' boundaries are the coordinate lines $\alpha=\alpha_{1,2}$. The solution of the stated problem will be sought in the form: 


$$
\begin{gathered}
U_{0}=x+i y+\sum_{n=1} a_{0 n} \mathrm{e}^{n\left(\alpha-2 \alpha_{1}+i \beta\right)}, \\
U_{1}=\sum_{n=1}\left[a_{1 n} \mathrm{e}^{n\left(\alpha-2 \alpha_{1}\right)}+b_{1 n} \mathrm{e}^{-n \alpha}\right] \mathrm{e}^{i n \beta}, \\
U_{2}=\sum_{n=1} b_{2 n} \mathrm{e}^{i n \beta-n \alpha} .
\end{gathered}
$$

The Fourier coefficients $a_{j n}$ and $b_{j n}$ in Eq. (7) are uniquely determined by taking into account boundary conditions. In particular

$$
a_{0 n}=2 c(-1)^{n} \frac{\lambda_{n}-1}{\lambda_{n}+1}, \quad \lambda_{n}=\frac{\varepsilon_{1}}{\varepsilon_{0}} \frac{\delta_{n}+1}{\delta_{n}-1}, \quad \delta_{n}=\frac{\varepsilon_{2}+\varepsilon_{1}}{\varepsilon_{2}-\varepsilon_{1}} \mathrm{e}^{2 n\left(\alpha_{2}-\alpha_{1}\right)} .
$$

The coefficients $a_{1 n}$ and $b_{1,2 n}$ have a similar form. For simplicity we do not write down explicit equations for these coefficients.

\subsection{The Maxwell-Garnett theory}

Let us now discuss the influence of the cylinder eccentricity on the effective characteristics of the medium. First, we consider the main principles of the Maxwell-Garnett theory for the set of identical inclusions randomly arranged in the matrix. Each of these inclusions consists of $n$ dielectric layers with permeabilities $\varepsilon_{i}, i=1,2, \ldots, n$. The effective dielectric constant $\varepsilon_{\text {eff }}$ can be defined from:

$$
\langle\mathbf{D}\rangle=\varepsilon_{e f f}\langle\mathbf{E}\rangle,
$$

where $\mathbf{D}$ is the electric displacement and the symbol $\langle\cdot\rangle$ denotes averaging of corresponding values. If we introduce quantities $f_{j}$ characterizing volume part of the $j$-th layer and $f_{0}=1-\sum f_{j}$ for the matrix, then the average electric displacement and electric field are given by:

$$
\langle\mathbf{D}\rangle=\sum_{j=1}^{n} f_{j} \varepsilon_{j}\left\langle\mathbf{E}_{\mathbf{j}}\right\rangle+f_{0} \varepsilon_{0} \mathbf{E}_{\mathbf{0}}, \quad\langle\mathbf{E}\rangle=\sum_{j=1}^{n} f_{j}\left\langle\mathbf{E}_{\mathbf{j}}\right\rangle+f_{0} \mathbf{E}_{\mathbf{0}} .
$$

By the linearity of the electrostatic problem, the values of average electric field strength are proportional to the external electric field $\left\langle\mathbf{E}_{\mathbf{j}}\right\rangle=p_{j}\left\langle\mathbf{E}_{\mathbf{m}}\right\rangle$ with coefficients of proportionality $p_{j}$. According to Eqs. (9)-(10) the expression for the effective dielectric permittivity is approximately given by:

$$
\varepsilon_{e f f}=\frac{\sum_{j=1}^{n} f_{j} \varepsilon_{j} p_{j}+f_{m} \varepsilon_{m}}{\sum_{j=1}^{n} f_{j} p_{j}+f_{0}} .
$$

For $n=1$ Eq. (11) is known as the Maxwell-Garnett formula [5]. More accurate results can be obtained with the T-matrix method [13]. In the case of concentric circles and $n=2$ we have [7]

$$
\begin{aligned}
& p_{1}=\frac{2 \varepsilon_{m}\left(\varepsilon_{1}+\varepsilon_{2}\right)}{\left(\varepsilon_{1}+\varepsilon_{2}\right)\left(\varepsilon_{0}+\varepsilon_{1}\right)+\left(r_{2} / r_{1}\right)^{2}\left(\varepsilon_{1}-\varepsilon_{2}\right)\left(\varepsilon_{0}-\varepsilon_{1}\right)}, \\
& p_{2}=\frac{4 \varepsilon_{m} \varepsilon_{1}}{\left(\varepsilon_{1}+\varepsilon_{2}\right)\left(\varepsilon_{0}+\varepsilon_{1}\right)+\left(r_{2} / r_{1}\right)^{2}\left(\varepsilon_{1}-\varepsilon_{2}\right)\left(\varepsilon_{0}-\varepsilon_{1}\right)} .
\end{aligned}
$$

It is possible to define the polarizability of a two-layer system as a $p=\left(p_{1} f_{1}+p_{2} f_{2}\right) /\left(f_{1}+f_{2}\right)$. Exactly this quantity is included in Eq. (11) for the effective dielectric constant. After performing numerical averaging of the series (7) and taking into account [12]

$$
\mathrm{d} x \mathrm{~d} y=\frac{c^{2}}{(\cosh \alpha+\cos \beta)^{2}} \mathrm{~d} \alpha \mathrm{d} \beta,
$$

we find the polarizability $p$. The dependencies of $p$ on the distance between the cylinders centers and relative dielectric constants $\varepsilon_{1} / \varepsilon_{0}$ are shown in Fig. 2.

From these graphs follows that if the distance between the cylinders centers $d$ is not too large, then the polarizability $p(d)$ is close to its value $p(0)$ for the concentric cylinders. Significant changes in polarizability have place only in the case where the outer and inner circles are almost touching each other. The proximity of 


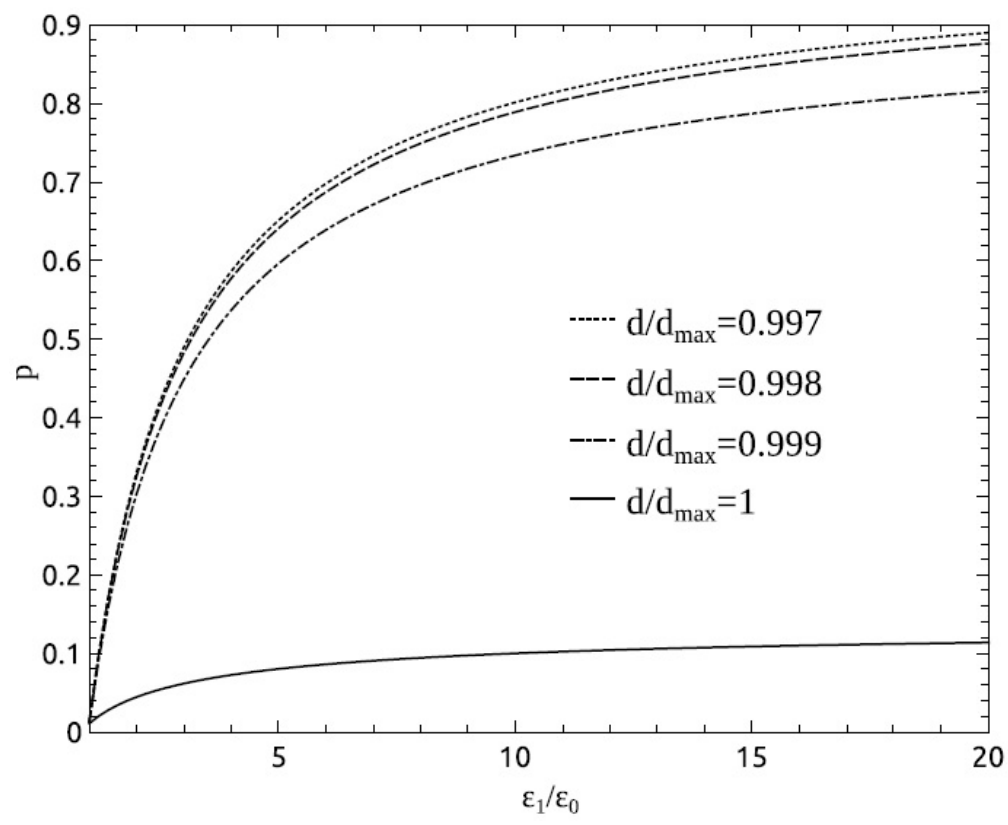

FIG. 2. The dependence of the polarizability $p$ on the ratio $\varepsilon_{1} / \varepsilon_{0}$ for the different relative distance $d$ between the cylinders centers

the cylinders boundaries leads to a noticeable decrease in the polarizability. The polarizability dependence on the dielectric constant of the inner cylinder is shown in Fig. 3.

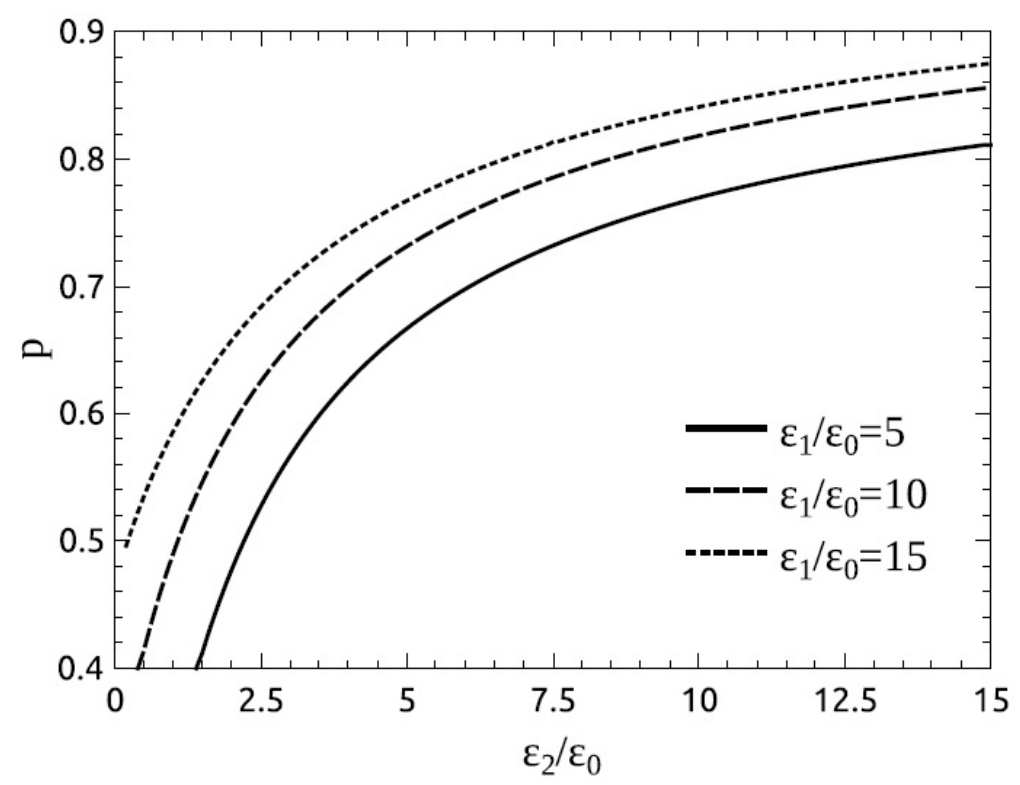

FIG. 3. The dependence of the polarizability $p$ on the ratio $\varepsilon_{2} / \varepsilon_{0}$

\section{Conclusion}

We have presented a new approach to describe the effective characteristics of a periodic set of eccentric cylinders. If the distance between the axes of the cylinder is small $\left(d / d_{\max }<0.997\right)$, the effective dielectric constant depends only on volume fractions of fillers. In this case it is possible to use the Maxwell-Garnett approximation. In turn, if the cylinders are almost touching each other $\left(d / d_{\max }>0.003\right)$, then the MaxwellGarnett method cannot be employed and the effective dielectric constant depends on the interaxial distance $d$. This 
dependence allows one to control the permittivity of composites. Perhaps, such a mechanism is realized in living cells. The cell nucleus might move inside the cell volume [14] and change its thermal and electrical conductivity when approaching the cell surface.

Also note that the dependence of the properties of eccentric cylinders on $d$ specifies the need for major modifications of classical averaging methods as Maxwell-Garnett, Bruggeman, etc. In these techniques, the effective dielectric constant depends only on the specific volume of the composite component. In more complex cases, however, the surface area of each component also can be taken into account (the Maxwell-Wagner effect). The change in the effective dielectric constant, as discussed in this paper, occurs at identical volumes and surface areas of each component. In addition, in our opinion, the angles of the edges and vertices at the boundaries of the various components need to be entered in the generalization of the formula for the effective permittivity of composites. Since no assumptions about the reality or positivity of cylinder dielectric constants $\left(\varepsilon_{1}\right.$ and $\left.\varepsilon_{2}\right)$ have been made, the resulting expressions are applicable for calculation of the metal cylinders with a complex permittivity and cylinder of metamaterials. It is also important also to emphasize, that the solution of piezoelectricity equations for eccentric circular fibrous composites under the generalized anti-plane shear deformation [15] may be constructed in precisely the same manner.

\section{Acknowledgements}

The research leading to the results exposed has received funding from the Government of Russian Federation, Grant 074-U01.

\section{References}

[1] Lagarkov A. N., Matytsin S. M., Rozanov K. N., Sarychev A. K. Dielectric properties of fiber-filled composites. J. Appl. Phys, 1998, 84(7), P. 3806-3814.

[2] Dang Z. M., Yuan J. K., Zha J. W., Zhou T., Li S. T., Hu G. H. Fundamentals, processes and applications of high-permittivity polymer-matrix composites. Progress in Materials Science, 2012, 57(4), P. 660-723.

[3] Alekseev A. V., Predtechenskiy M. R. Aluminum foil reinforced by carbon nanotubes. Nanosystems: Physics, Chemistry, Mathematics, 2016, 7(1), P. 185-189.

[4] Khodke M. R., Joshi S. V. An investigative study on application of carbon nanotubes for strain sensing. Nanosystems: Physics, Chemistry, Mathematics, 2016, 7(4), P. 755-758.

[5] Garnett J. C. M. Colours in metal glasses, in metallic films, and in metallic solutions. II Philosophical Transactions of the Royal Society of London, Series A, 1906, 203, P. 237-288.

[6] Rayleigh L. LVI. On the influence of obstacles arranged in rectangular order upon the properties of a medium. The London, Edinburgh, and Dublin Philosophical Magazine and Journal of Science, 1892, 34(211), P. 481-502.

[7] Zhang H., Shen Y., Xu Y., Zhu H., Lei M., Zhang X., Xu M. Effective medium theory for two-dimensional random media composed of core-shell cylinders, Opt. Comm., 2013, 306, P. 9-16.

[8] Starkov I. A., Starkov A. S. Effective parameters of nanomatryoshkas. Journal of Nanophotonics, 2016, 10(3), P. 033503-033503.

[9] Grinberg G. A., Selected Topics in the Mathematical Theory of Electrical and Magnetical Phenomena. Acad. of Sci. USSR, Leningrad, $1948,733 \mathrm{pp}$.

[10] Chaumet P. C., Dufour J. P. Electric potential and field between two different spheres. Journal of electrostatics, 1998, 43(2), P. $145-159$.

[11] Balagurov B. Y. On the polarizability of a pair of cylinders in a transverse electric field. Technical Physics, 2003, 48(8), P. 945-951.

[12] Lebedev N. N., Skalskaya I. P., Uflyand Y. S. Worked Problems in Applied Mathematics. Dover, New York, 2010, 420 pp.

[13] Kiselev A. D., Plutenko D. O. Light scattering of Laguerre-Gaussian beams: near-field structures and symmetries. Nanosystems: Physics, Chemistry, Mathematics, 2016, 7(2), P. 349-370.

[14] Bunge R. P., Bunge M.B., Bates M. Movements of the Schwann cell nucleus implicate progression of the inner (axon-related) Schwann cell process during myelination. The Journal of Cell Biology, 1989, 109(1), P. 273-284.

[15] Xiao J., Xu Y., Zhang F. A generalized self-consistent method for nano composites accounting for fiber section shape under antiplane shear. Mechanics of Materials, 2015, 81, P. 8194-8200. 